\title{
EL PAPEL DE LA UFSM Y SU PROGRAMA DE POSGRADO EN EXTENSION RURAL EN EL MERCOSUR
}

\author{
Pedro de Hegedus ${ }^{1}$
}

\section{RESUMO}

Este artigo se propõe a rememorar, em perspectiva histórica e pessoal, o importante papel exercido pelo PPG em Extensão Rural da UFSM no intercâmbio de pesquisas, experiências, métodos, ações, projetos, publicações e pessoas relacionadas às temáticas da Extensão e Desenvolvimento Rural no âmbito do Mercosul.

Palavras-chave: desenvolvimento rural, extensão rural, Mercosul.

\section{THE ROLE OF UFSM AND GRADUATE PROGRAM RURAL EXTENSION IN MERCOSUR}

\begin{abstract}
This article aims to look back on historical and personal perspective, the important role played by PPG in Rural Extension of the UFSM in the exchange of research, experiences, methods, actions, projects, publications and people related to the themes of Extension and Rural Development under Mercosur.
\end{abstract}

Keywords: rural development, rural extensión, Mercosur.

Cuando me propusieron escribir, acerca de la importancia que ha tenido desde una perspectiva histórica el Programa de Posgrado en Extensión de la Universidad Federal de Santa Maria (UFSM), en ocasión de festejar los cuarenta años, obviamente acepté rápidamente por la amistad y los lazos fraternos que me unen con esta Universidad, y en particular, con el Departamento de Educación Agrícola y Extensión Rural (DEAER). Tuve el honor de ser profesor visitante en ese posgrado en los primeros años del actual siglo, y por eso puedo atestiguar acerca del proceso transcurrido, y desde esa perspectiva reflexionar, tomando en cuenta la realidad de la Extensión Rural en las últimas décadas en nuestros países.

Si bien resulta difícil dar cuenta pormenorizada de toda la riqueza, quisiera compartir con los lectores algunas reflexiones que me parecen centrales y que hacen al papel jugado por la UFSM en las últimas décadas. En los momentos más difíciles de la Extension en los países de nuestro continente, hablo de la década del

\footnotetext{
${ }^{1}$ Graduado em Agronomia pela Universidad de La Republica. Mestrado em Extensión Agricola pela lowa State University of Science and Technology. Doutorado em Extensión Agricola pela lowa State University of Science and Technology. Professor adjunto a Universidad de La Republica. E-mail: phegedus@adinet.com.uy
} 
90, el aporte de un conjunto de cuatro instituciones en el Mercosur fue clave para mantener viva la enseñanza y la investigación en Extensión. Las mismas son: a) el INTA y la Universidad de Rio Cuarto en Argentina, b) la Universidad Federal de Santa Maria en Brasil, y c) la Universidad de la Republica en Uruguay (Facultades del Área Agraria y el Servicio Central de Extension y Actividades en el Medio).

Si me apuran, digo que en las últimas décadas del siglo pasado y principios de la actual, los trabajos que en conjunto se hacían entre estas Instituciones, ayudaron mucho a mantener la llama (mística) encendida en el Mercosur, en torno a la necesidad de trabajar desde la academia para aportar y poder contar en la realidad con Servicios de Asistencia Técnica y Extensión Rural (SATER). Posiblemente en Argentina y Brasil, a diferencia del continente, la situación, si bien difícil, fue en esas épocas relativamente menos grave, debido a la fuerte presencia pública institucional diseminada en la amplitud de los territorios, que amortiguaba los golpes e intentos de destrucción. En Uruguay, por ser un país de menor tamaño, esta posibilidad no existió. No solo se debilitaron fuertemente los SATER, también se redujeron a su mínima expresión la formulación de políticas públicas tecnológicas amplias, de las cuales los SATER son una parte. Esto afectaba las capacidades del país en ciencia, tecnología e innovación (PEIXOTO, 2011). Los SATER trabajan en el uso social del conocimiento, y en la articulación de los ámbitos y grupos que se vinculan con su generación.

Hablamos de la década del 90, en donde se llevó a cabo en America Latina un modelo neoliberal de fuerte ajuste macroeconómico y desmantelamiento de la estructura del Estado como agente de desarrollo, ubicándose estas políticas en las antípodas de lo que una vez se denominó el modelo del Estado de Bienestar aplicado en el continente (MANZANAL, 2006).

Por eso para mí, cuando regrese del doctorado en USA, gracias a una beca Fulbright, me encontré en 1993 con un país en donde se había agudizado el cierre de los servicios públicos de Extensión, y ni se mencionaba esta palabra (y se inventaban en forma creativa otros nombres para sustituirla); todo se había reducido a un problema de marketing (como captar la demanda). En noviembre de 1995 fui invitado para "una palestra", a la UFSM, y lo que veía era otra realidad, que alentaba la esperanza de liberar energías capaces de generar impulsos como para llevar adelante procesos alternativos en mi país. Ahí conocí por primera vez a Hugo Vela, y la gente del DEAER. Ellos organizaban el Primer encuentro de Extensión Rural del Cono Sur. Me encontré con profesionales y académicos de la Extensión de los países vecinos y Paraguay. Que brecha había con Uruguay! Estos encuentros se continuaron en otros, y reitero, toda esa dinámica fue tremendamente enriquecedora y promovedora de acciones, como la creación del Grupo de Salto, llamado así, porque nos juntábamos los países en la ciudad de Salto, un punto cercano a todas las instituciones vinculadas.

Estrictamente hablando, la primera vez que me contacté con la UFSM, fue en Montevideo, en 1990, en ocasión de recibir a una "turma" de estudiantes del posgrado, dirigida por los profesores José Renato Duarte Fialho y Gustavo Martin Quesada (profesor que había trabajado en USA con el mismo Everett Rogers!), que visitaban diferentes experiencias en el país (los procesos alternativos que hablaba antes!). Ya me había sorprendido en ese entonces esta presencia tan activa y en estos temas! 
E. Rogers ${ }^{2}$, antes mencionado, es el autor central de uno de los dos paradigmas existentes en Extensión: el paradigma de la difusión. Podemos asociar este paradigma, que surge a mediados del siglo pasado, a la Revolución Verde, a la teoría de la modernización, y a la estrategia de intervención de "transferencia de tecnología". La lógica central señala que el efecto de extensión (la acción de los servicios de extensión al inicio de un programa "capacitando" a los productores) más la acción de difusión (la acción de los productores que han adoptado lo recomendado y por "contagio" trasmiten al resto esta conducta), permiten asegurar que la tecnología propuesta se adoptará masivamente (HEGEDUS, 2013).

El paradigma alternativo que surge como respuesta es el de Paulo Freire: La educación problematizadora. La relación entre el técnico y productor es horizontal, en donde el técnico facilita el proceso. La clásica transmisión de conocimientos (educación bancaria) cede paso al fenómeno creativo de compartir la experiencia en el terreno de la intersubjetividad. Hay una comunicación de ida y vuelta, ambos, "el que sabe y el que no sabe" se educan entre sí, mientras se establece un diálogo que apunta al "empoderamiento" del educando. Para Freire enseñar exige una reflexión crítica sobre la práctica. Si logramos que el productor produzca su comprensión de lo aprendido, luego de reflexionar sobre lo que hacemos ( $\mathrm{y}$ la curiosidad ingenua se vuelve crítica), existe una verdadera situación de enseñanza aprendizaje (FREIRE, 2006).

Ahora bien, y volviendo al tema luego de estas breves referencias a los paradigmas, justicia es señalar que de las instituciones mencionadas, fue la UFSM la que tuvo el papel más destacado, en el sentido que percibió la importancia estratégica de generar los ámbitos, a partir del posgrado en Extensión (casi el único existente en la región en ese entonces), para que diferentes instituciones pudieran enriquecerse unas a otras y de la confluencia operativa de recursos y motivaciones poder avanzar en la construcción de propuestas desbloqueando al mismo tiempo los obstáculos. Este hecho del posgrado fue decisivo. En los cursos de este posgrado participaron décadas atrás jóvenes de Argentina y Uruguay que luego jugaron un papel destacado (pienso, sin agotar en absoluto la lista, en Gustavo Cimadevilla, Mario Costa, Humberto Tommasino, entre otros) trabajando en las temáticas vinculadas a Extension.

Ricardo Thornton realizó su post doctorado en la UFSM por los inicios del actual siglo, y de la tesis derivo un libro que fue el inicio de una secuencia importante de publicaciones de la dupla Thornton - Cimadevilla. Se llamó "La Extensión rural en debate: Concepciones, retrospectivas, cambios y estrategias para el Mercosur" (también aparece el título en portugués!). Recuerdo que en este libro realizamos un capitulo en conjunto con Hugo Vela, y otro con un estudiante de la Maestria en la UFSM, se trataba de Marcelo Porto Nicola (actualmente extensionista de EMATER).

Otro libro importante que ejemplifica este relacionamiento regional es "Extension: reflexiones para la intervención en el medio urbano y rural" $(\mathrm{H}$. Tommasino y $\mathrm{P}$. de Hegedus como editores y autores de capítulos, conjuntamente con los compañeros del DEAER y los de Argentina). Fue realizado en "parceria" entre la UFSM y la UDELAR, con el apoyo de una colonia de productores en Uruguay. Se presentó en el paraninfo de la UDELAR en Uruguay a sala repleta. Participaron las siguientes Autoridades de esa época: el Ministro de Ganadería, Agricultura y Pesca, José Mújica, el ex Pro Rector de Extensión, C. Rucks, el Rector

\footnotetext{
${ }^{2}$ Ver Rogers, E. M. y Shoemaker, F. F. Comunicación de innovaciones, un enfoque transcultural. México, CRAT, 1971, p. 385. Este libro resume el paradigma difusionista. La última edición es de 1985.
} 
de la UDELAR, J. Arocena, el Decano de la Facultad de Agronomía, F. Garcia, y el Decano de la Facultad de Veterinaria, G. Kramer. Posteriormente este libro obtuvo el Premio de la Academia Nacional de Veterinaria 2011- Ministerio de educación y Cultura, entregado en la sala de actos de la Facultad de Veterinaria el 23 de noviembre del 2011.

Docentes importantes también participaron en ese posgrado, como Miguel Vassallo, actualmente en la conducción del Instituto Nacional de Colonización en Uruguay. Y la UFSM tuvo un docente clave en la persona de Hugo Vela, que actuó en verdad como un excelente nodo articulador de las relaciones entre estas instituciones, conformando la red antes señalada. Hugo fue un entusiasta promotor de la vinculación de nuestros países con la UFSM y creo que en su recuerdo el mejor homenaje es continuar con esta sinergia!

Numerosos libros, proyectos, cursos, jornadas, encuentros, tesis, y otros mecanismos, posibilitaron entonces que desde la UFSM se avanzara en el (re)pensar del rol de la extensión y el asesoramiento técnico ante los nuevos escenarios de la ruralidad y los dilemas de la sustentabilidad, abarcando el cambio técnico y los procesos de desarrollo rural, asumiendo los errores y aciertos como aprendizaje, y sentando las bases para el establecimiento de nuevas políticas, que llegaron a partir de los inicios de este siglo con la presencia de nuevos Gobiernos en la región, que desarrollaron políticas en relación con la producción familiar y sectores vulnerables, abriendo las puertas de nuevo a la Extensión (luego de la década del 90).

Un gran mérito de esta red desde que se inició fue el de buscar obviamente una postura académica, sin renunciar a la autonomía y objetividad, a partir de la inserción en la realidad, dialogando con los actores que trabajaban en el propio terreno de las intervenciones. Este contacto no existía en el siglo pasado, a diferencia de lo que siempre aconteció en USA o Europa.

Un ejemplo paradigmático de la importancia de este posgrado de Extensión se relaciona con el enfoque agroecológico, que constituye en la actualidad uno de los seis enfoques de trabajo en Extensión existentes en America Latina (junto con transferencia de tecnología, capacitación y visitas, experimentación adaptativa, desarrollo de capacidades, y enfoque territorial) ${ }^{3}$. Algunos lo llaman directamente investigación-acción participativa. Si ubicamos a Caporal y Costabeber como los que desarrollaron teóricamente el enfoque, ellos fueron estudiantes de este posgrado en esa década del 90 , se formaron en la UFSM. Costabeber (lamentablemente fallecido y de quien tengo un gran recuerdo por su jerarquía técnica y calidad humana), luego de algunas décadas de trabajo en EMATER retornó a la UFSM como docente y trabajo en este posgrado.

Otro ejemplo de la importancia de este posgrado de Extensión es el espacio ganado por la Revista de Extensión Rural de la UFSM, que se publica desde 1993 al presente, y que es una de las mejores revistas en Extensión Rural que existen en America Latina y el mundo. No es poca cosa este esfuerzo! Y que importante es, para sistematizar intervenciones y plantear reflexiones de interés por su proyección estratégica. Bien sabido que esto es algo que cuesta mucho a los extensionistas, más propensos a la acción que al análisis que se deriva de la misma. En esta revista, de carácter trimestral y muy bien conceptuada, han escrito muchos autores de enorme valía, al respecto menciono por ejemplo en el No. 7 del año 2000 nada menos que a José Graziano da Silva. Los temas de la Revista son variados y relacionados al Desarrollo rural y la Extensión desde diferentes perspectivas.

\footnotetext{
${ }^{3}$ Resultado de una Consultoría efectuada para el BID (INE/RND). Evaluación de las reformas a los sistemas de extensión / transferencia de tecnología en America Latina (RG-K1316) en el 2013.
} 
Algunos tópicos repetidos: Agricultura familiar, regiones, cadenas productivas, sustentabilidad, gestión agrícola, márgenes de comercialización y estimaciones de costos, identidad de actores sociales, cooperativismo y asentamientos rurales, alternativas generadoras de renta, metodologías de Extensión y de prácticas pedagógicas, transición agroambiental, turismo, etc.

$\mathrm{Si}$ analizamos en las últimas décadas las formas de ver y entender a la Extensión en nuestro continente, hay como dos grandes miradas (que se derivan de las heterogeneidades existentes en aspectos históricos, económicos, culturales, políticos) que es necesario tener en cuenta. Tenemos la mirada del "Atlántico", conformada básicamente por Argentina, Brasil y Uruguay, y la mirada del "Pacífico", conformada en esencia por el resto de los países. En otros fenómenos también ocurre lo mismo. Por ejemplo en el cooperativismo (NEIMAN, 2009), Argentina, Brasil (principalmente el sur) y Uruguay presentan el mismo modelo, basado en la influencia de inmigrantes europeos, que llegaron aproximadamente a fines del siglo 19 y principios del siglo 20, y traían consigo la experiencia cooperativa europea. Los restantes países de América del Sur y Central desarrollan un modelo a partir de procesos promovidos por los Gobiernos (Alianza para el Progreso en la década del sesenta, reformas agrarias, etc).

Volviendo a la Extensión, si ya de por si el debate semántico acerca de que es o no es la Extensión, ocupó mucho tiempo y alimentó discusiones sin llegar a consensos (tengo la sensación de que este debate fue muchas veces paralizante de la acción), también esta diferente forma de entender la Extensión contribuyó a la dificultad de acuerdos generalizados. La primera mirada del "Atlántico", pone el acento en la institucionalidad pública para entender a la Extensión desde esa perspectiva, mientras que la segunda mirada, del "Pacífico", la analiza y la entiende desde el mercado. Ambas miradas enriquecen y no implican juicios de valor. Para la primera mirada, esa red multi-institucional que menciono en este artículo ha sido y es, una usina intelectual de enorme importancia para sistematizar, analizar y aportar elementos desde la reflexión académica a las políticas. En la segunda mirada hay varios núcleos importantes, ubico por ejemplo debido a su importancia al RIMISP en Chile (y sus aportes al enfoque territorial).

Quienes estamos en la primera mirada, simplemente porque aquí vivimos, tenemos que seguir en este camino de interacciones en conjunto multiinstitucionales, de participar en la red (y no enredados), siempre sin desvirtuar la rigurosidad científica, y con sensibilidad ante los cambios y procesos que acontecen, para lo cual se necesita flexibilidad para adecuarse a las circunstancias cambiantes y capacidad de proyección para que las estrategias puedan contribuir eficazmente al logro de los objetivos.

Finalmente, quiero cerrar este trabajo con una reflexión. La gran lección aprendida en nuestros países es que el Estado no se puede retirar de las políticas vinculadas al Desarrollo Rural y la Extensión. Esto significa que debe existir una estrategia pública para estas funciones, que en definitiva implican el desarrollo rural entendido desde una visión amplia, que apuesta a la inclusión y a la innovación procurando la articulación de esfuerzos de una pluralidad institucional pública y privada diseminada en los territorios. Esta estrategia debe ser de largo plazo, para facilitar la integración de las diferentes políticas e instrumentos, ya que la Extensión aislada, difícilmente tendrá impacto. La producción familiar y asalariados rurales, o temas que se caracterizan por su transversalidad (calidad, inocuidad, medio ambiente) necesitan de la Extensión pública, con lineamientos establecidos y con fuentes de financiamiento públicas que hay que consolidar. El mercado por sí solo no da las respuestas. Si el Estado no está presente, siempre existirá un proceso de difusión natural y cierto uso de la información, pero pueden no coincidir con el 
interés de la sociedad y excluir a los sectores de la producción familiar y asalariados, de su acceso. La Extensión es un bien público y cabe al Estado la responsabilidad de instrumentar las políticas que permitan ese acceso. Las formas de lograrlo pueden ser varias, pero la responsabilidad es intransferible e implica que el Estado es un actor en el proceso.

\section{REFERENCIAS}

FREIRE, P. Pedagogía de la autonomía. São Paulo: Editorial Paz e Terra, 34. ed., 2006, p. 135.

HEGEDUS, P. Estrategias de extensión: Los agricultores familiares y su adaptación al cambio climático en territorios seleccionados del Cono Sur. Informe final de consultoría. IICA - PROCISUR, 2013, p. 60.

MANZANAL, M. Regiones, territorios e Institucionalidad del desarrollo rural. In: MANZANAL, M.; NEIMAN, G.; LATTUADA, M. Desarrollo rural: organizaciones, instituciones y territorios. Buenos Aires: Ediciones Ciccus, p. 449.

NEIMAN, G. Desarrollo rural y cooperativismo: desafíos, oportunidades y estrategias. FAO-INTA. Roma, Italia, 2009, p. 133.

PEIXOTO, C. Política tecnológica para el sector agropecuario. Informe consultoría BID, 2011, p. 84. 